\title{
Treatment Planning of Dental Implants in the Anterior Maxilla; Risk Assessment and Review of Soft Tissue along with Bone Preservation and Augmentation Techniques for Successful Clinical Outcomes
}

\author{
Nkem Obiechina \\ Pannu Dental Associates, 40880 Fremont Boulevard, Fremont, CA
}

\begin{abstract}
The anterior maxilla continues to present with high potential risk for esthetic failure, and as a result, there is a clear need for modifications that would allow for natural-looking restorations that are harmonious with the rest of the mouth. A number of changes in protocol for placing implants such as using a restorative-driven protocol, the performance of a risk assessment and addressing factors that could compromise esthetics such as deficiencies in bone and soft tissue using bone and soft tissue grafts to ensure adequate tissue volume are necessary for dental implant overall success in the anterior maxilla. Understanding of timing with regard to implant placement has also contributed to achieving esthetic success in the region. This article reviews modifications made in implant placement in the esthetic zone and how they can contribute to functional and esthetic success in the anterior maxilla.
\end{abstract}

Key Words: Dental implants, Risk assessment, Mouth

\section{Introduction}

The anterior maxilla requires careful consideration during treatment planning dental implant placements due to unique conditions that are present [1]. When people smile the crowns of their anterior teeth and some soft tissue is usually visible, it is, therefore, essential that implant restorations in the anterior maxilla be harmonious with adjacent natural teeth so as not to distract from a person's smile. Because the goal is to provide dental implants and restorations that are aesthetically pleasing and in harmony with a patient's natural dentition and adjacent restorations, careful treatment planning and risk assessment is needed in other to achieve successful outcomes.

Having adequate bone and soft tissue dimensions, adequate dental implant positioning in the apico-coronal, mesio-distal and buccolingual dimensions, as well as correct angulations of implants, are important factors to ensuring overall esthetic and functional success around implants. In assessing bone and soft tissue dimensions around implants, the goal of surgical therapy is a harmonious gingival margin without major changes in tissue height, maintaining intact papilla and preserving soft tissue contours [2]. The goal is that during surgical therapy, implants are placed in positions that allow dental implant restorations in the anterior maxilla to blend effectively with adjacent natural dentition [2-5].

The anterior maxilla has traditionally in the past been the region in the mouth that has been of major esthetic concern for dentists that are placing and restoring dental implants [6]. The potential reasons that deem teeth in the anterior maxilla hopeless can range from vertical root fractures, recurrent failure of root canals, trauma, dental caries and periodontal disease all of which can leave the region deficient in bone and soft tissue support. Bone defects that can occur in dental implant sites include intra-alveolar defects, dehiscence, fenestration, horizontal and vertical ridge defects, [7] while defects in soft tissue include deficiency in volume and quality of tissue around the dental implant site (Figure 1).

Hammerle and Tarnow classified the reasons for deficiencies in bone and soft tissue around dental implant sites to occur due to trauma from tooth extractions, systemic conditions that can lead to incomplete bone and tooth formation, periimplantitis, mechanical overload, anatomic preconditions, thin soft tissue as well as lack of keratinized tissue [7].
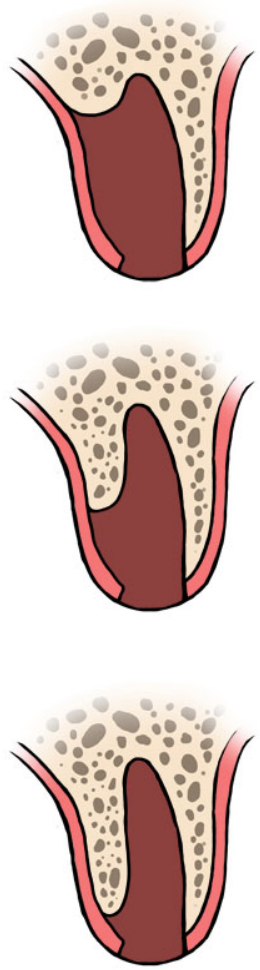

Figure 1. Dehiscence type defect.

While overall success rate in the anterior maxilla has been found by multiple studies to be very high [6,8-10] these deficiencies can have a detrimental impact on positioning, angulation and having adequate tissue support to mask restorative components [11]. As a result, the failure to address them can lead to the potential for esthetic failures in the region. 
During treatment planning dental implants in the anterior maxilla, a restorative-driven protocol has been recommended involving placing dental implants in surgical positions that will result in optimal implant restoration [3-5]. To accomplish this involves the placement of dental implants in sites with adequate bone volume and soft tissue contours present. This usually involves the use of bone grafts and soft tissue augmentation to address the deficiency in bone and soft tissue to create optimal sites for implant placement. The goals of restorative-driven implant placement in the anterior maxilla involves success in four components; placement of dental implants in optimal positions with adequate bone and soft tissue support, correcting any discrepancies in soft tissue contour and form and ensuring adequate tissue support for facial aspect and embrasure areas [2,5].

Other components include use of provisional restorations to contour soft tissue around implants in preparation for definitive implant restoration, and finally placement of a permanent implant restoration that is in harmony with adjacent teeth and surrounding soft tissue with no major changes in color or contours $[2,5]$.

\section{Concepts in Maxillary Anterior Treatment Planning}

In treatment planning maxillary anterior implants it is essential for implant success to adhere to four major concepts which include: dental implant placement in appropriate positions in the mesiolingual, distolingual and apico-coronal dimensions, implant placement with the correct angulation, choosing the appropriate dental implant size, avoiding use of excessively large implant sizes for maxillary anterior implants, and ensuring that there is adequate soft tissue present for development of dental implant soft tissue contours and interproximal papilla [2].

In assessing dental implant positioning, Buser et al. characterized areas around edentulous sites for implant placement as "comfort" and "danger" zones [2]. Demarcation of these areas allows identification of locations where implant positioning could lead to potential esthetic compromise "danger zones" and areas where dental implant placement would be optimal for restorative success "comfort zones" (Figure 2).

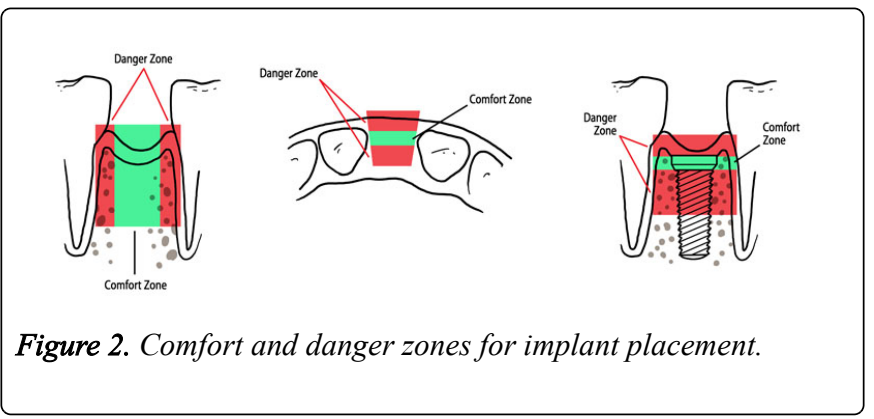

To position dental implants in the mesiodistal dimension, the goal is to have a minimum of $1.5 \mathrm{~mm}$ distance between adjacent roots of natural teeth and dental implants, and a minimum of $3 \mathrm{~mm}$ between adjacent dental implants [2]. Danger zones are areas that are close to adjacent teeth and implants. Failure keeps to the recommended distance can result in resorption of the bone crest to the implant site causing reduced papilla height [2].

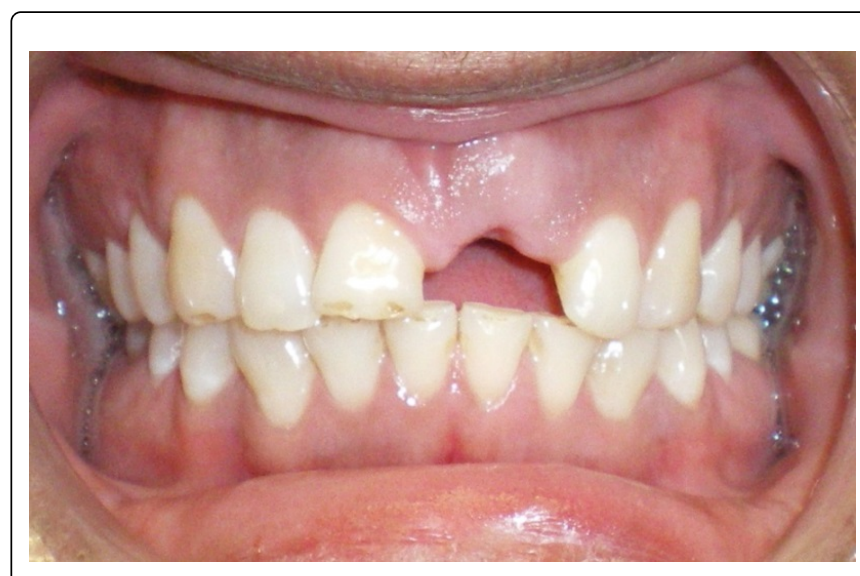

Figure 3. Initial patient presentation for papilla preservation technique.

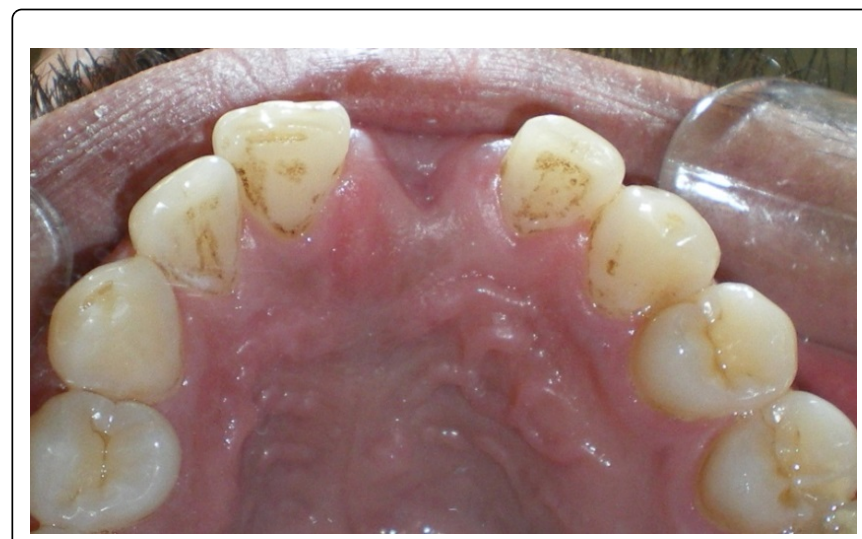

Figure 4. Initial presentation [2].

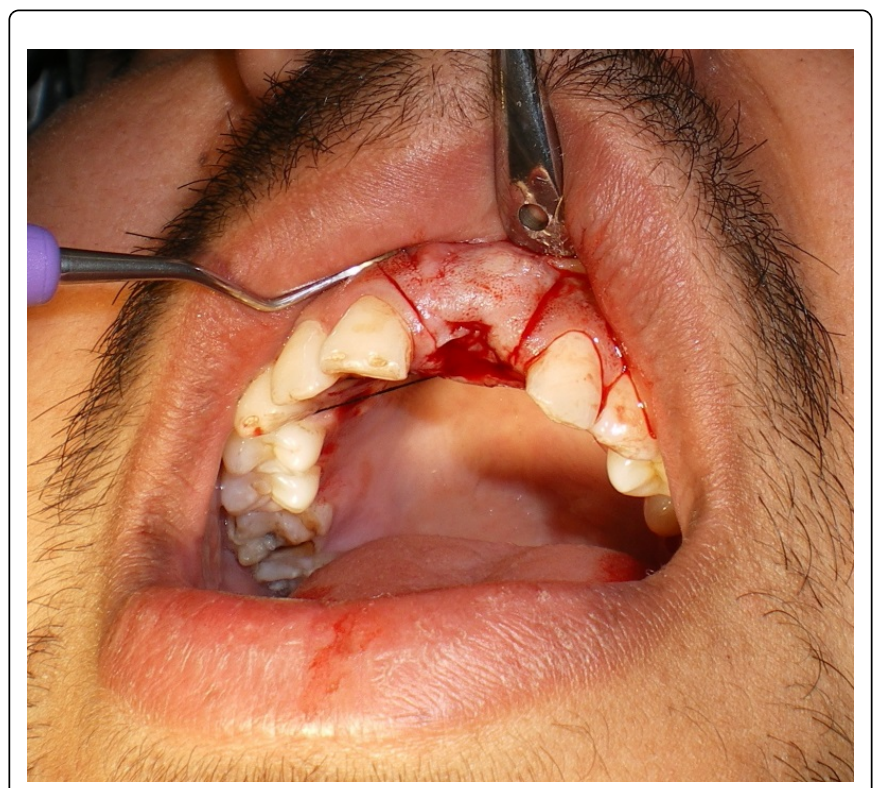

Figure 5. Papilla preservation incision.

Placing implants within optimal positions in the buccolingual dimension requires that the implants should be placed $1 \mathrm{~mm}$ palatal to an imaginary line at the point of emergence profile of adjacent teeth to the implant site. 
Implant placement facial to this site is a potential danger zone and can result in loss of facial bone.

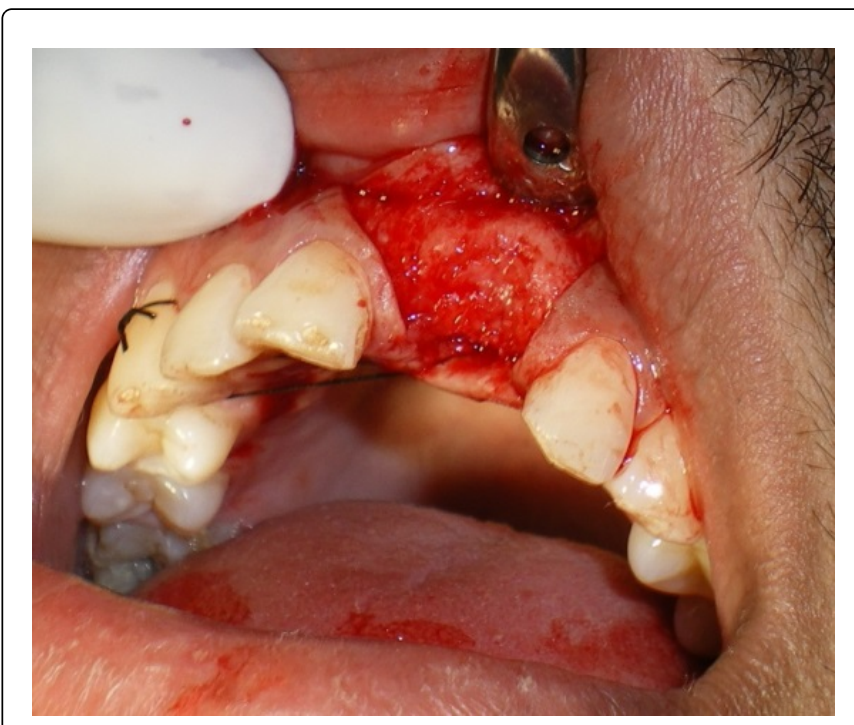

Figure 6. Flap.
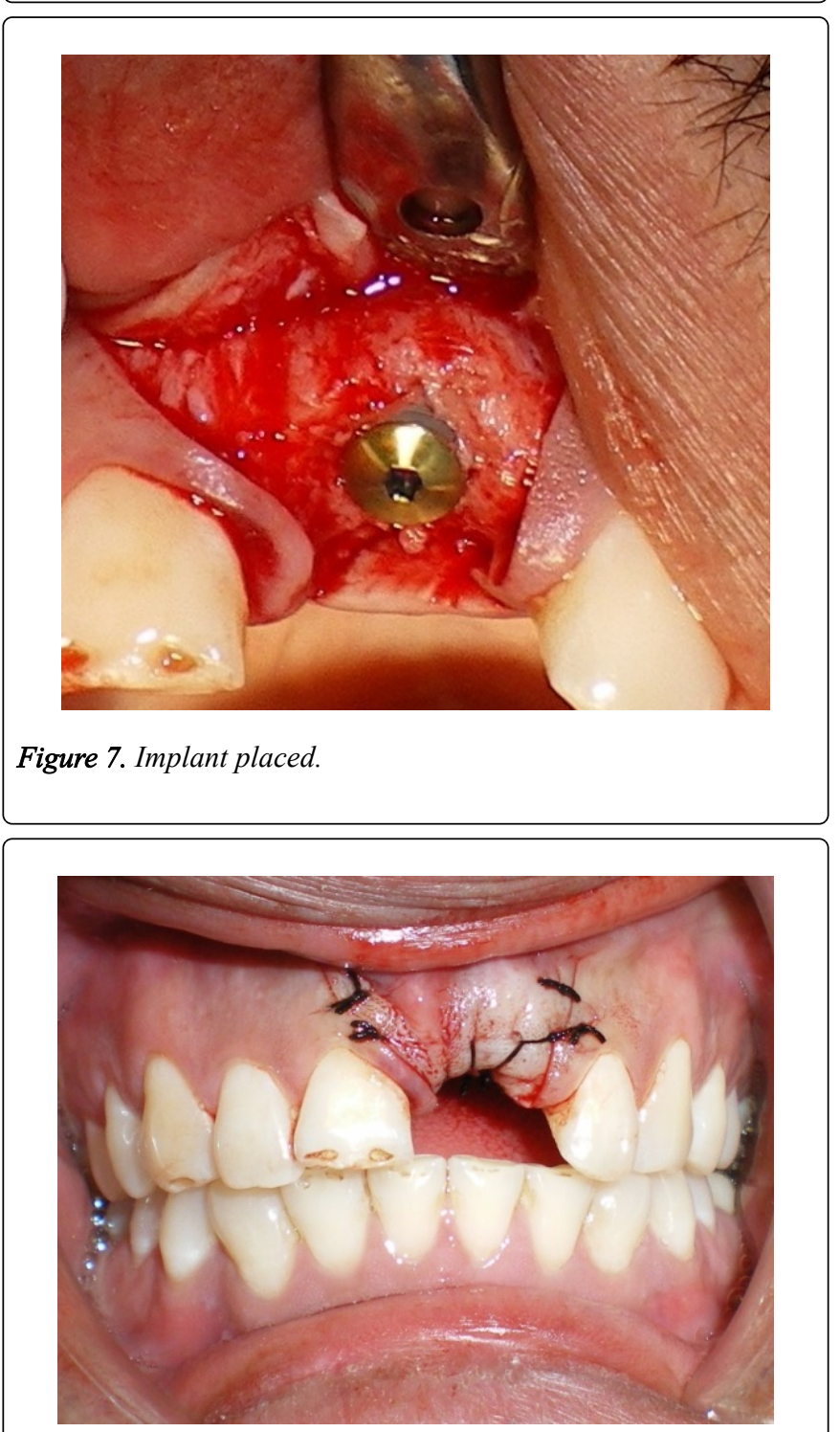

Figure 8. Sutures.
Placement of dental implants more than $2 \mathrm{~mm}$ palatal to this line can result in a potential ridge lap restoration with impeded oral hygiene assess [2].

The apico-coronal dimension typically involves dental implant placement $2 \mathrm{~mm}$ from the midfacial gingival margin of the planned restoration. Apico-coronal danger zone involves dental implant placement that is more than $3 \mathrm{~mm}$ from the gingival margin of the planned implant restoration [2]. The goal is to stay within the advised distance to prevent problems with potential bone loss, restorations that are too long, and failure to develop complete papilla inter-proximally (Figures 3-8).

When treatment planning maxillary anterior dental implants, the goal is to identify potential causes that can be able to impede the esthetic success and try to address them prior to dental implant placement. To accomplish this involves the use of a risk assessment which lists the potential risks that can affect maxillary anterior dental implant esthetic success allowing them to be corrected prior to dental implant placement $[2,3,5]$.

\section{Performing a Preoperative Risk Assessment}

In performing a pre-operative risk assessment, a medical history is completed which allows screening for uncontrolled medical conditions, radiation therapy, prolonged use of bisphosphonates and corticosteroids and smoking habits $[5,12]$. A chief complaint is obtained, and dental history with information to assess a patient's overall expectations to ensure that they are realistic is also completed. This is then followed by an extraoral exam that evaluates general parameters such as facial symmetry, midline, the orientation of occlusal plane, presence of lip support, assessment of smile width and smile line [13].

In assessing lip support and smile line, a patient that presents with low smile line shows $75 \%$ or less of their anterior teeth crowns when they smile, this makes them have a low esthetic risk during dental implant restoration. Patients with medium smile lines show $75 \%-100 \%$ of their anterior teeth when they smile and usually their interproximal papilla while those with high smile lines show all the crowns of their anterior teeth as well as $2 \mathrm{~mm}$ or more of gingival tissue [13] (Figures 9-11).

Patients that have low and medium smile lines tend to show less of their teeth and gingival tissue on a full smile, and present with less esthetic risk than those with high smile lines who show more of their gingival tissue when they smile hence any changes in the color or thickness of tissue can prove problematic.

An intra-oral examination is also completed as part of the pre-operative risk assessment. This allows for an assessment of the overall gingival and mucosal tissue. This is followed by an occlusal assessment which allows assessment for parafunctional habits and bruxism, as well as evaluation of the interocclusal space in the edentulous site. This usually is accomplished after obtaining study models, utilizing mounted casts and diagnostic wax-ups.

This is then followed by obtaining appropriate X-rays and then by a restorative and periodontal exam to identify caries, 
endodontic lesions or active periodontal disease that might be proximal to the implant site and initiate a treatment plan for treatment prior to dental implant therapy $[2,5]$. CT scan Xrays are also ordered for a three-dimensional assessment of the anatomy of the potential implant site [2].
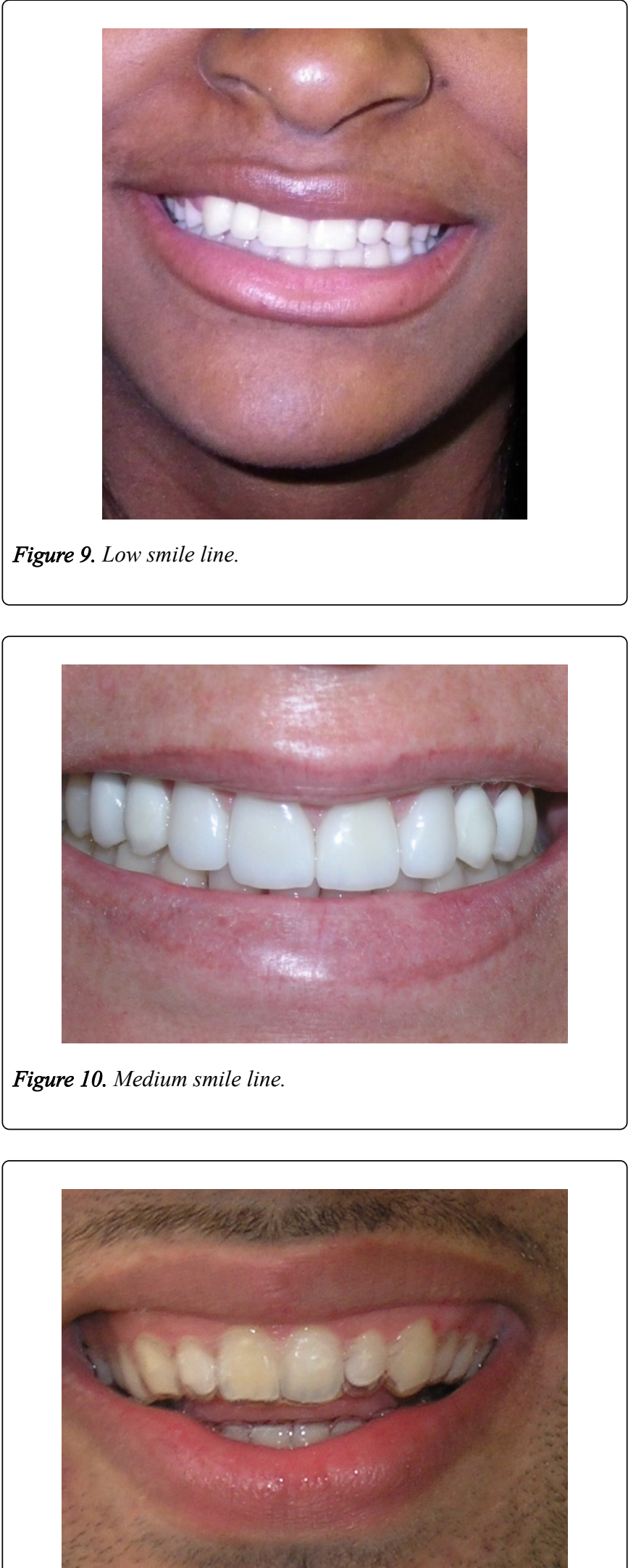

Figure 11. High smile line.

\section{Performing an Anatomic and Surgical Risk assessment}

In other to avoid factors from contributing to the aesthetic failure of maxillary anterior implants, Buser et al. recommend completing an anatomic and surgical risk assessment to ensure that deficiencies in bone and soft tissue and other potential risk are addressed [2]. CT scan x-rays are usually combined with bone sounding in other to evaluate bone width, height, length and density at the dental implant site. CT scan X-rays can also be able to detect changes in bone anatomy such as dehiscence and fenestrations, as well as concavities in bone [2].

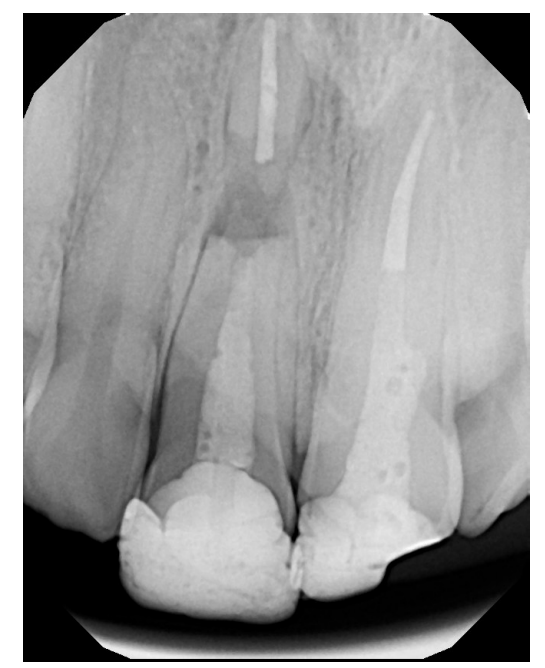

Figure 12. Procedure on a patient with high smile line Initial $X$ ray.

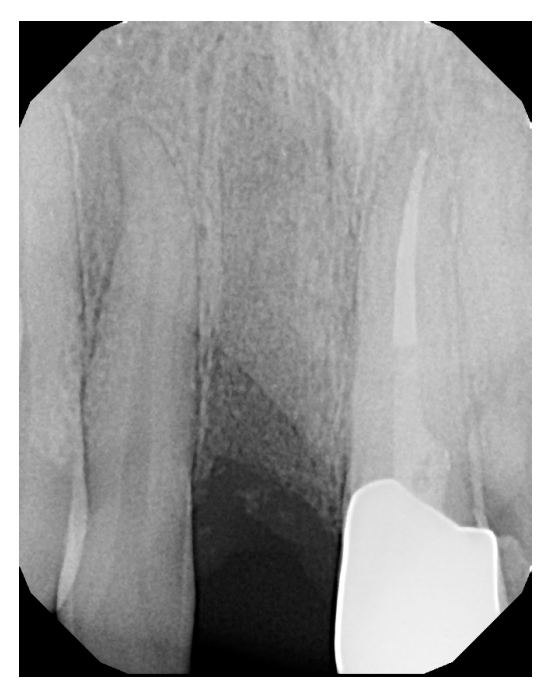

Figure 13. Procedure on a patient with high smile line extraction X-ray.

Anatomic factors which can contribute to the esthetic failure of implants in the anterior maxilla include biologic width violation, excessive loss of interproximal bone, inadequate facial bone thickness, placement of dental implants into sites with bone deficiency and concavity, and implant placement in sites with thin soft tissue phenotype [2]. In 
assessing biologic width, its violation can have the impact of causing inflammation and bone loss similarly to around teeth [14-16]. Around implants biologic width was found to be composed of the sulcus/peri-implant sulcular epithelium, periimplant junctional epithelium, oral epithelium and connective tissue consisting of a distance of about $3.08 \mathrm{~mm}$ with usually a range of 3-4 mm, unlike biologic width of about $2.04 \mathrm{~mm}$ around teeth, its violation can result in potential for bone loss $[14,15]$.

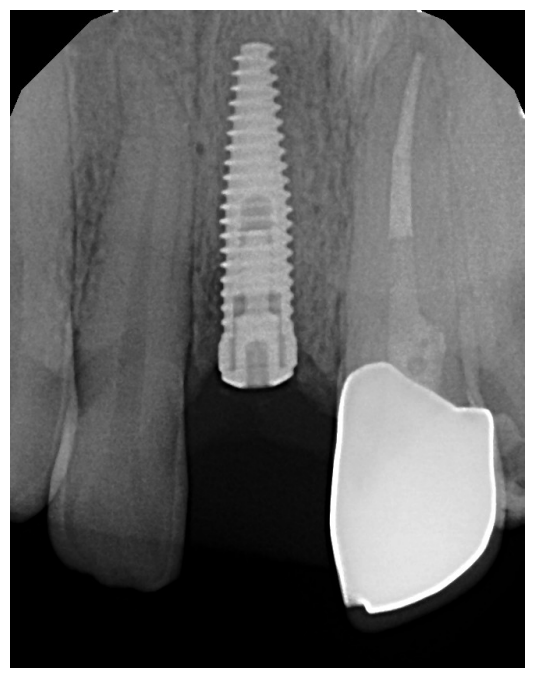

Figure 14. Implant X-ray.

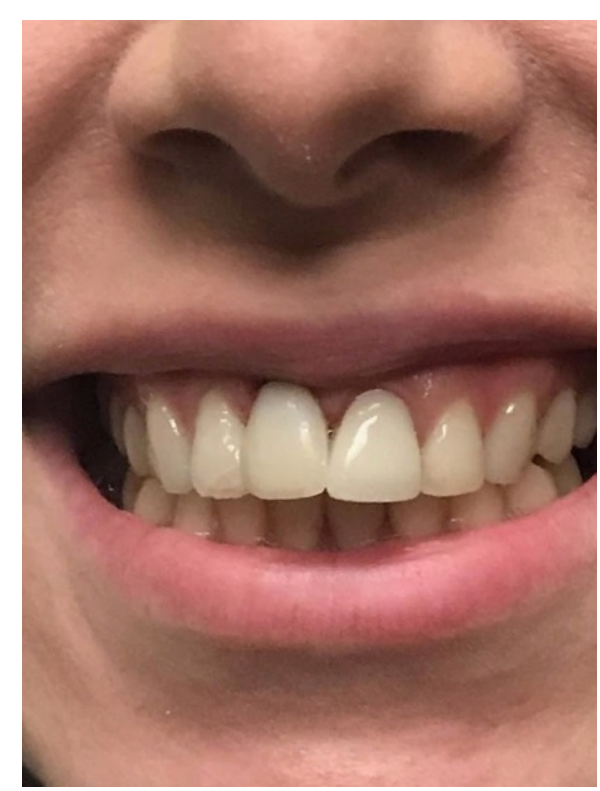

Figure 15. Patient restored showing smile line.

Potential iatrogenic causes of esthetic dental implant failures include poor implant positioning in one or more of the three dimensions, use of dental implants that are too wide in the anterior maxilla, improper angulation of dental implants, improper mesio-distal proximity to adjacent teeth, and apical location of the micro gap between the implant and abutment interphase leading to bone resorption [2]. Most iatrogenic causes of dental implant failure can be prevented during dental implant planning with using CT scans to assess implant sites, and surgical guides during dental implant placement, as well as by ensuring that there is adequate bone for implant positioning in all dimensions and by selecting standard implant diameters in the anterior maxilla rather than excessively wide implants $[2,5,17]$.

In the assessing smile line, patients that have high smile lines are at greater risk of esthetic failure because they show more of their gingival tissue. It is therefore important that there is an adequate amount of soft tissue be present as well as that the gingival color is the same as that of the adjacent teeth, and that implant patients have good oral hygiene habits. Presence of gingival inflammation or thin gingival tissue contour significantly affects esthetics in the maxillary anterior area, so when this exists, it is essential that it should be treated prior to implant restoration [2] (Figures 12-15).

For patients that present with a thin facial tissue or thin gingival tissue phenotypes, use of soft tissue grafts help to enhance gingival contours and thickness. Thicker gingival phenotypes tend to be less prone to recession and are better at withstanding inflammation. Use of soft tissue grafts that increase the thickness of tissue as well as placement of the dental implants at a more palatal location will allow for the ability to better improve esthetic outcome by improving tissue thickness around implants with thin facial gingival phenotypes $[2,5,18]$. Having thicker gingival phenotypes is also essential for maintaining oral hygiene around dental implants as well as preventing gingival inflammation or gingival recession $[5,19]$.

\section{Bone and Soft Tissue Preservation during Implant Placement}

To avoid problems in dental implant positioning in the mesiodistal, facio-lingual or apico-coronal dimensions, having adequate bone support is essential prior to dental implant placement. Bone loss and concavities in the bone should be addressed with bone grafts prior to dental implant placement, for sites with thin facial bone, a combination of bone grafts and soft tissue grafts is recommended to help inthe formation of a thick tissue phenotype [5]. Use of surgical guides is essential to preventing problems in positioning and angulation in the anterior areas where even the slightest error can compromise esthetics especially for patients with medium and high smile lines. Prior to anterior dental implant placement, impressions are taken for surgical guides, and if guided technology is being utilized they are combined with information from CT Scans for the fabrication of surgical guides using CADCAM technology.

When evaluating the interproximal papilla area, the goal is to have complete papilla height coverage around the anterior dental implant in order to ensure that no dark triangles occur due to incomplete soft tissue in the interproximal area. The interpapillary bone crest determines the amount of interpapillary coverage that will occur, typically when the distance is less than $5 \mathrm{~mm}$ there is a high chance that complete interpapillary tissue will be present around implants, but as the distance increases in millimeters to $6 \mathrm{~mm}$ the chances of getting complete papilla height decreases significantly [20]. Salama et al. identified the term "predictable papilla length" as the achievable papilla length in the maxillary anterior sextant measured as the most coronal interproximal height 
(IHB) immediately adjacent to a tooth or dental implant after surgical or restorative therapy [20]. The distance between implants is $4.5 \mathrm{~mm}, 5 \mathrm{~mm}$ for natural teeth, and $5.5 \mathrm{~mm}$ between adjacent dental implants [20]. At dimensions above these distances, the risk increases that the papilla might not be formed completely and dark triangles could occur (Figures 16-18).

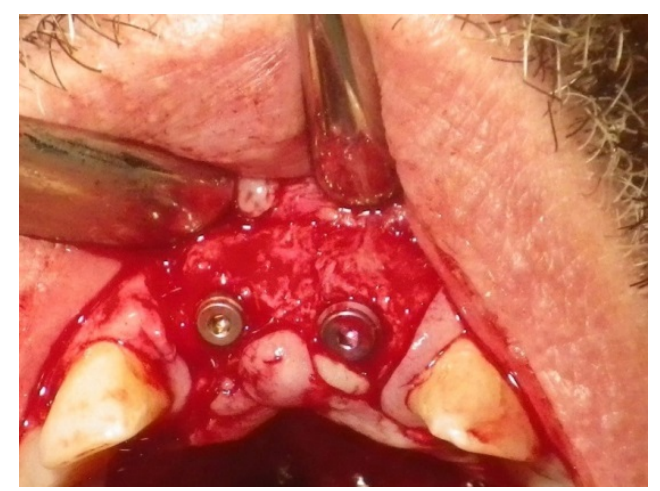

Figure 16. Implant positioning with 2 implants.

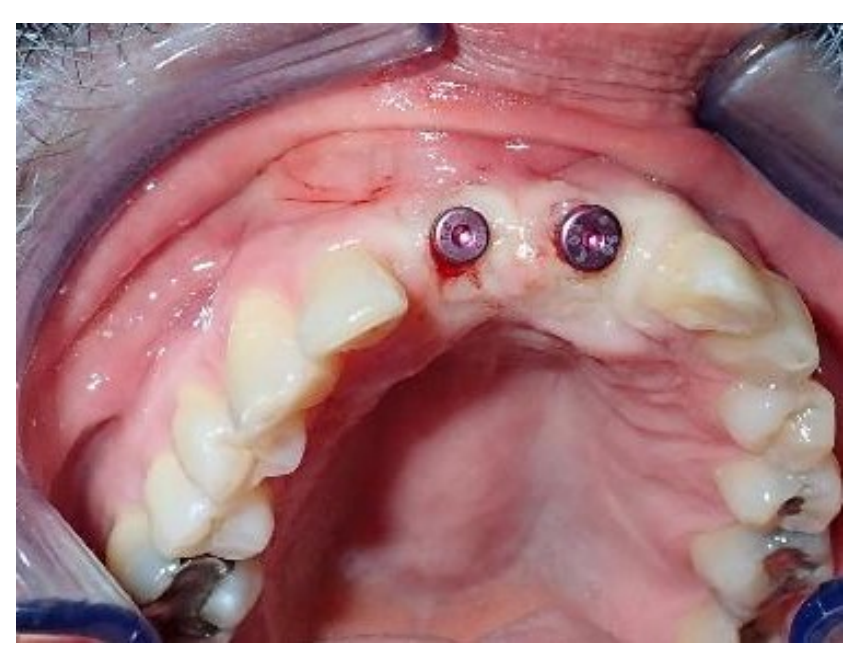

Figure 17. Abutment in place.

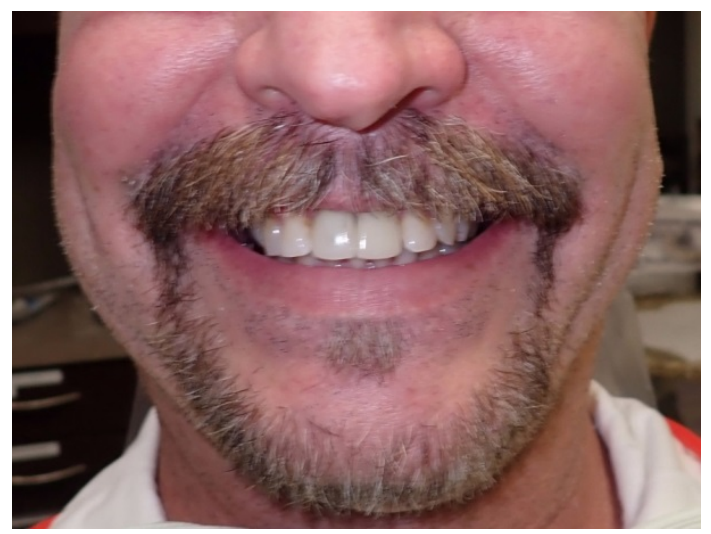

Figure 18. Implants restored.

The soft tissue around implant restorations plays a major role in the overall health and esthetics around dental implant restorations. When thin gingival tissue is present, it can result in un-esthetic outcomes such as gingival recession showing implant components, gingival inflammation resulting from inadequate keratinized tissue around the implant site, as well as the potential for peri-implantitis [21,22]. Failure of the interproximal papilla to form could also occur which can result in esthetic complications especially for patients with high smile lines. A number of studies have stressed the importance of using soft tissue augmentation to improve tissue contours around dental implants as well as to increase the width of keratinized tissue around dental implants [5,11,19-23].

When patients present with thin gingival phenotypes at implant sites or potential for insufficient soft tissue exists, use of soft tissue graft have been used to improve tissue thickness, keratinized tissue width as well as gingival contours, and are highly recommend for use in the anterior zone prior to or in conjunction with dental implant placement and restoration [21]. When there is a failure to achieve adequate soft and bone volume by augmentation, use of other methods might be utilized such as the use of pink porcelain to mask tissue loss, as well as adjustment of the contact point to have an illusion of interproximal tissue might be necessary [23]. These techniques can present a compromise to esthetics so as much as possible measures should be taken to prevent needing to use them.

During the surgical phase, modifications can be made to help preserve tissue in the interproximal areas when placing dental implants [24]. Use of papilla preservation flaps can be able to allow implant placement without significantly affecting interproximal tissue and include techniques such as flapless technique, papilla preservation techniques, and Ushaped flap design as well semilunar incisions [25]. The goal is preserving interproximal tissue in order to ensure adequate tissue height around dental implant restorations. Kan et al. identified that the most effective way to recreate papilla is to take measures to prevent its loss and loss of underlying bone at the time of tooth removal [26]. They recommend the use of immediate implant placement in the maxilla with augmentation when needed and use of properly contoured provisional restorations to preserve papilla height and tissue contours [26].

Greenstein and Tarnow recommended the use of papilla preservation techniques during surgical therapy in the maxilla to prevent recession of papilla and potential formation of dark triangles [27]. They recommend a flapless technique when adequate keratinized tissue and bone are present in the site, and use of a surgical design avoiding reflecting the papilla by including vertical incisions and extending the flap $1 \mathrm{~mm}$ from the interpapillary area on either side [27]. The failure to include the papilla in the flap helps to keep an intact blood supply to the papillary area as well as prevents potential bone loss and tissue recession that could occur when a flap containing the papilla is raised [27].

\section{Procedures for Bone and Soft Tissue Augmentation}

Defects that occur in bone and soft tissue can be horizontal or vertical in nature, as a result, require surgical therapy to correct tissue deficiency. In augmenting implant sites using 
bone and soft tissue grafts, horizontal defects tend to have a more predictable result than vertical augmentation. Socket preservation techniques are usually treatment planned with for immediate or delayed implant placement. When socket preservation is being done with immediate placement, it is recommended that the extraction would be atraumatic and that implant placement occurs with tissue augmentation, as well as a provisional restoration to continue to shape soft tissue contours in preparation for the permanent restoration [26].

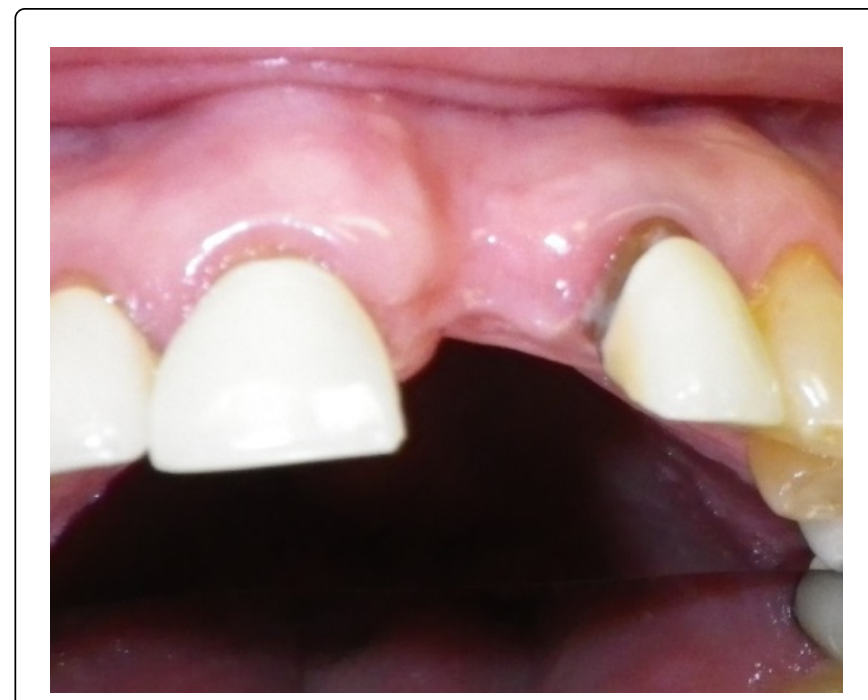

Figure 19. Site with bone and soft tissue defect.

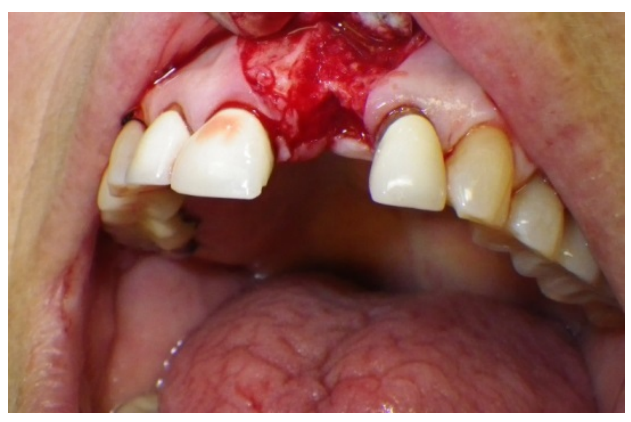

Figure 20. Bone defect.

Kazor et al. reviewed socket preservation techniques with delayed implant placement and advocated three socket preservation techniques [28]. The first involved flapless atraumatic extraction, and socket preservation using either autogenous bone particles, demineralized bone, or synthetic bone combined with an absorbable collagen matrix, or barriers that are either absorbable, non-absorbable or acellular dermal grafts [27]. He also advocated the use of the Bio-Cal technique developed by Schlar [29] involving atraumatic extraction, socket curettage, and socket grafting using deproteinized bovine hydroxyapatite graft, with an absorbable collagen membrane, tissue cement, followed by the use of an ovate pontic to contour soft tissue in the site for implant placement [28,29]. An additional modified socket preservation technique involves atraumatic extraction, socket curettage, socket grafting with Demineralized Bone Allografts (DFDBA) and free soft tissue graft secured with interrupted sutures [28]. The surgical site is allowed to heal for 6 months to enhance bone support and ideal soft tissue contours prior to dental implant placement [28].

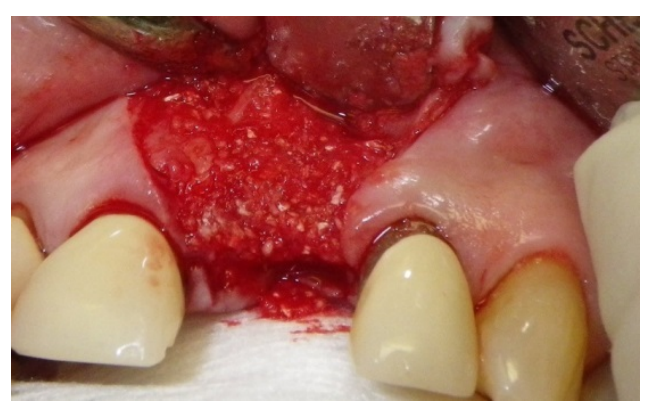

Figure 21. Bone graft in place.

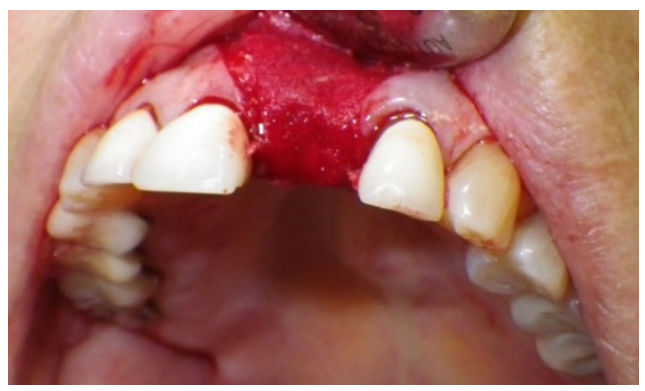

Figure 22. Membrane in place.

Use of forced tooth eruption using orthodontics to extrude hopeless teeth that are scheduled for extraction was described by Salama et al. [30]. The goal has been using forced eruption of teeth to help to develop alveolar height and preserve soft tissue in order to correct ridge height deficiencies prior to dental implant placement [30,31]. It offers the advantage of promoting the growth of hard and soft tissue before extraction of teeth [31]. The PDL around the natural tooth is the key factor that allows bone and soft tissue augmentation prior to extraction [30,31]. Not only does orthodontic extrusion increase the dimensions of bone, but it also moves the free gingival and interdental papilla more coronally while the location of the mucogingival junction stays the same [32] Indications include periodontal vertical defects, site preservation or enhancement before dental implant placement, and sites that have uneven osseous or gingival levels [30,31] Recommendations for use of extrusion include sites that do not have active periodontal disease, usually extruded teeth need endodontic therapy prior to extrusion [31] It usually involves a slow extrusion process of between 1-2 mm per month with orthodontic forces parallel to long axis of the tooth using orthodontic brackets or elastic thread for movement [31]. Potential drawbacks for the procedure include increased treatment time with technique, and potential to lose interproximal papilla with excessive force, as well as it is not usually recommended for labially inclined teeth [28].

Augmentation of the ridge when local defects occur laterally can occur using autografts, particulate grafts, or block grafts combined with barriers to regenerate lost bone support. The process of guided bone regeneration involves the use of barrier membranes to form physical barriers that prevent in the growth of competing for non-osteogenic cells 
into the membrane space allowing bone regeneration to occur (Figures 19-24).

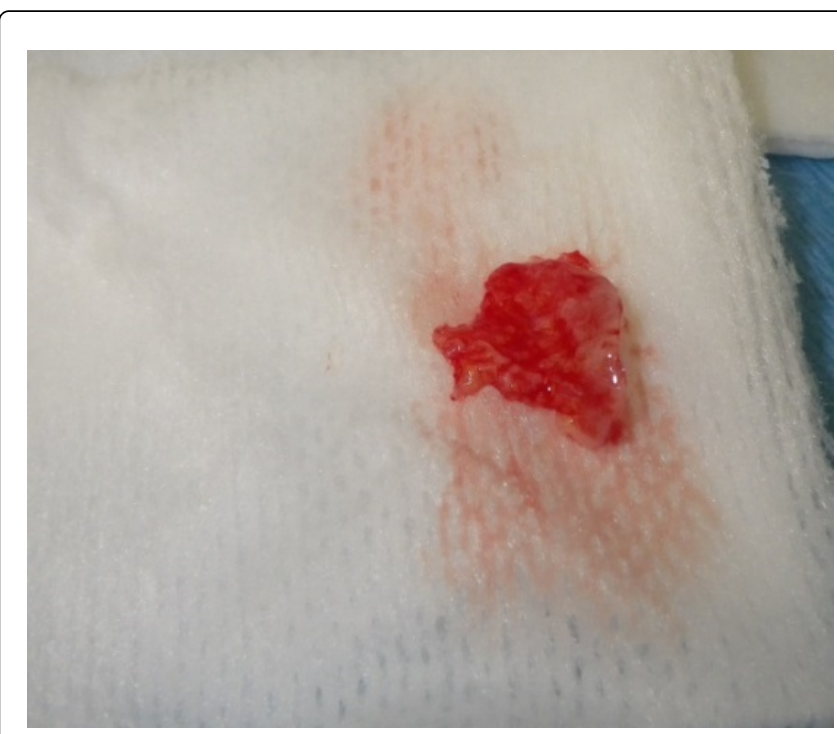

Figure 23. The free soft tissue graft.

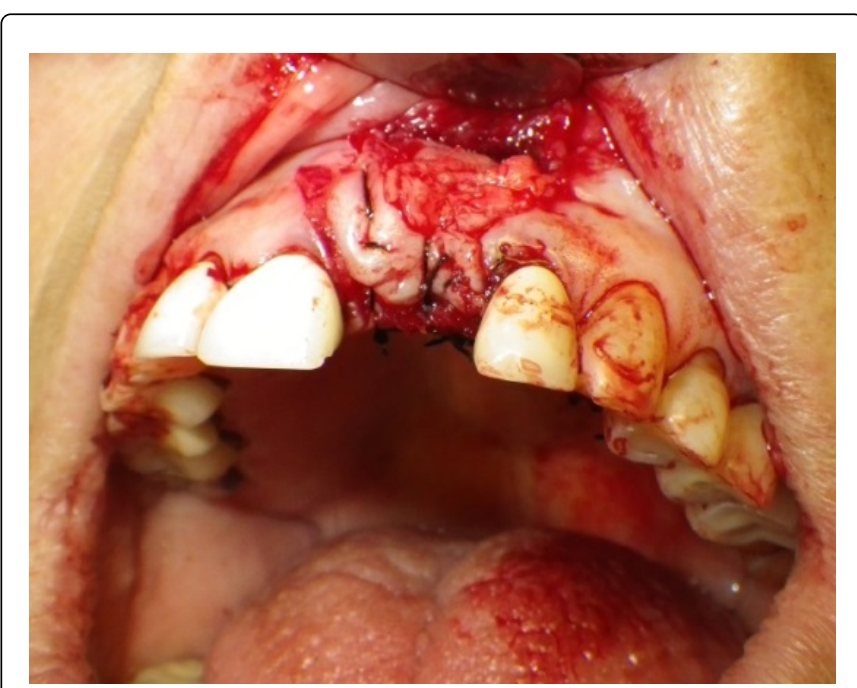

Figure 24. Free soft tissue graft sutured in place.

Buser et al. [33] evaluated the use of autogenic bone grafts combined with E-PTFE membranes for lateral augmentation in 40 edentulous patients and showed a post-enlargement gain of $3.5 \mathrm{~mm}$ to $7.1 \mathrm{~mm} \mathrm{[33].} \mathrm{They} \mathrm{concluded} \mathrm{that} \mathrm{augmentation}$ using autografts and ePTFE membranes is a predictable way to regenerate bone and augment the ridge laterally [33]. Other techniques for horizontal augmentation include use of splitcrest technique involving expansion of the ridge that requires an increase in bone diameter of 2-5 $\mathrm{mm}$ of bone [34].

Vertical augmentation of alveolar ridge sites are usually less predictable than horizontal augmentation, and methods for vertical augmentation include only bone grafts using autogenic bone, distraction osteogenesis involving use of gradual controlled displacement of two separated bone fragments with new bone cells growing in the gap, block grafts and staged or simultaneous guided bone regeneration using membranes and titanium mesh [34-36] To ensure that adequate soft tissue closure is present, various techniques can be combined with the augmentation procedure such as coronally positioned flap combined with connective tissue grafts, pouch and envelope technique using sub-epithelial connective tissue grafts, use of coronally positioned flap and acellular dermal grafts as well as free gingival grafts [28].

\section{Classifying Soft and Hard Tissue in the Anterior Maxilla}

Palacci et al. classified tissue loss in the maxilla as vertical or horizontal [37]. According to the classification, vertical loss around the papilla area can be divided into Class I to IV while horizontal tissue loss is classified as Class A-D. Class I refers to an intact or slightly reduced papilla, Class II deals with limited loss of papilla of about $50 \%$, Class III refers to a severe loss of dental papilla while Class IV involves a complete absence of dental papilla [37]. In assessing horizontal loss tissue loss in the anterior maxilla, Class A refers to intact or slightly reduced buccal tissue, Class B involves limited loss of buccal tissue, Class $\mathrm{C}$ deals with severe loss of buccal tissue, while Class D involves extreme loss of tissue in combination with limited attached mucosa [37]. When assessing maxillary anterior tissue loss, based on the classification of the tissue loss, the timing and type of implant therapy required is determined.

While patients that present with Palacci Class I-A classification can be able to undergo implant placement including immediate dental implant placement with minimal surgical complications expected, patients that present with Class III and Class IV classification would typically be prepared for delayed implant placement with bone and soft tissue grafting [37]. For patients that present with Class IIB classification, soft tissue augmentation is combined with implant placement, for patients that present with III C combination, they recommend delayed placement with bone augmentation techniques combined with soft tissue graft and use of provisional restorations to contour soft tissue. For patients that present with Type classification, Palacci et al. advocate ensuring that the patients are fully aware of the fact they present with severe bone resorption and soft tissue collapse with high potential for esthetic compromised clinical outcome prior to starting their implant therapy [37]. In addition to multiple bone grafting and soft tissue augmentation, they recommend the potential need for orthodontic extrusion, segmental mastectomy, distraction osteogenesis as well as the potential need for use of pink porcelain to mask lost tissue support [37].

\section{Timing of Dental Implant placement}

Funato and Salama et al. evaluated the timing of dental implant placement and stressed its importance to overall implant success in the anterior maxilla [2]. They divided the timing of implant placement into three classes, Class 1, involving the extraction, immediate placement using either incisionless technique or mucoperiosteal flap with osseous augmentation, guided bone regeneration or connective tissue and soft tissue allografts.

Class 2 timing includes early dental implant placement 6-8 weeks after extraction to allow soft tissue healing. Guided bone regeneration would be done at the time of extraction or 
during dental implant placement [2]. Class 3 involves delayed implant placement at 4-6 months after extraction with preservation of the maxillary alveolar ridge using grafting or guided bone regeneration which is usually done at the time of extraction or implant placement [2].

Teeth that have been scheduled for extraction due to having a hopeless prognosis would have immediate dental implant placement if there is no bone compromise and if the interproximal height of bone (IHR) of adjacent teeth is within $4.5 \mathrm{~mm}$ in order to allow complete papilla formation, 1 distance more than that could result in incomplete papilla fill $[2,20,38]$. When the IHR distance is more than $4.5 \mathrm{~mm}$, it is recommended to utilize a delayed approach rather than immediate dental implant placement [2]. Use of orthodontic extrusion is also recommended to improve vertical tissue dimensions prior to immediate implant placement [2]

An advantage of immediate implant placement in the anterior maxillary area is the ability to preserve bone and soft tissue support in the implant site, and prevent loss of 3-4 mm of bone that can occur within the first six months after tooth extraction [39]. The goal is atraumatic extraction, and immediate placement combined with a provisional restoration which is designed to place lateral pressure on the tissue around the dental implant preserving the shape and location of the soft tissue, preventing tissue collapse and retaining the soft tissue emergence profile [39]. This allows for a definitive restoration that blends harmoniously with adjacent teeth.

In recommending implant therapy based on the classification proposed by Funato A and Salama $\mathrm{M}$ et al., Class 1 involves intact buccal bone with thick soft tissue phenotype and immediate placement of dental implant utilizing a flapless technique is usually completed [2]. Class 2 involves intact buccal bone with thin gingival phenotype which requires immediate placement with soft tissue graft or a secondary soft tissue graft after placement. Class 3 involves buccal bone with bone loss that may have implant placement with bone augmentation and guided bone regeneration depending on the extent of buccal plate loss. If extensive, then they recommend a delayed approach [2]. Class 4 involves delayed dental implant placement with bone and soft tissue augmentation in which CT scan X-rays and three-dimensional planning with surgical guides are needed for dental implant success [2].

\section{Conclusion}

The anterior maxilla presents unique challenges that make implant placement in the region complex. The goal is achieving esthetic and functional success with maxillary anterior restorations and careful planning is essential for success. Incorporating timing, risk assessment, as well as the use of restorative-driven implant placement has been essential to improved accuracy and the overall efficacy of dental implant placement in the maxillary anterior area. Studies incorporating an understanding of bone and soft tissue dimensions have also been essential to overall implant success in the esthetic zone, and as further research is concluded they will continue to have an even greater impact on improving overall esthetic and functional implant success in the anterior maxilla.

\section{Reference}

1. Kuchler U, Van Arx T. Horizontal augmentation in conjunction with or prior to implant placement in the anterior maxilla: A systematic Review. International Journal of Oral and Maxillofacial Implants. 2014; 29: 14-24.

2. Buser D, Martin WC. Optimizing esthetics for implant restorations in the anterior maxilla: anatomic and surgical considerations. International Journal of Oral and Maxillofacial Implants. 2004; 19: 43-61.

3. Funato A, Salama M Ishikawa T, Garber DA, Salama H. Timing, positioning, and sequential staging in esthetic implant therapy: a four dimensional perspective. International Journal of Periodontics and Restorative Dentistry. 2007; 27: 313-323.

4. Ishikawa T, Salama M, Funato A, Kitajama H, Moroi H, et al. Three dimensional bone and soft tissue requirements for optimizing esthetic results in compromised cases with multiple implants. International Journal of Periodontics and Restorative Dentistry. 2010; 30: 502-512.

5. Levine RA, Guy HB, Cochran DL. Soft tissue augmentation procedures for mucogingival defects in esthetic sites. International Journal of Oral Maxillofacial implants. 2014; 29: 155-185.

6. Al-Sabbagh M. Implant in the esthetic zone. Dental Clinics of North America. 2006; 50: 391-407.

7. Hammerle CHF, Tarnow D. The etiology of Hard and soft tissue deficiencies at dental Implants: A narrative review. Journal Clinical Periodontology. 2018; 45: S267-S277.

8. Gao EF, WeiHong H, Park JC, Pang KM, Kim SK, et al. Bone level implants placed in anterior maxilla, an open label single arm observational study. Journal of Periodontal implant Science . 2017; 47: 312-327.

9. Buscerlecher D, Furhauser R, Haas R, Watszek G, Mailath $\mathrm{G}$, et al. Long term implant success at the academy of implantology: 8 year follow up and risk analysis. Journal of Periodontal Implant Science. 2014; 44: 102-108.

10. Ghardoudi AMR, Homayouni A, Rokn AR, Kia F, Kharazefard MJ, et al. Frequency of dental implants placed in the esthetic zone in dental clinic of Tehran University: A descriptive study. Journal of Dentistry of Tehran University of Medical Sciences. 2015; 12: 906-912.

11. Schneider D, Grunder U, Ender A, Hammerle CHF, Jung RE. Volume gain and stability of perimplant tissue following bone and soft tissue augmentation. 1 year results from a prospective cohort study. Clinical Oral Implant Research. 2011; 22: 28-37.

12. Al-sabbagh M. Implants in the esthetic zone. Dental Clinics of North America. 2006; 50: 391-407.

13. Bal A, Dugal R, Shah K, Mudaliar U. Principles of Esthetic evaluation for anterior teeth, Journal of Dental and Medical Sciences. 2016; 15: 28-38.

14. Rezai EZ, Kadkhidadeh M, Gholamin P, Amid R, Passaneri E, et al. Biologic width around dental implants and updated review. Journal of Dental Material Technology. 2016; 5: 68-81.

15. Atsuta I, Ayukawa Y, Kondo R, Oshiro W, Matsuura Y, et al. Soft tissue seal around dental implants based on a 
histological interpretation. Journal of Prosthodontic Research. 2016; 60: 3-11.

16. Yeung SCH. Biologic basis for soft tissue management in implant dentistry. Australian Dental Journal. 2008; 53: S39-S42.

17. Deeb GR, Deeb JG. Soft tissue grafting around teeth and dental implants. Oral and Maxillofacial surgery clinics of North America. 2015; 27: 425-448.

18. Ioannou AL, Kotsackis GA, Michale MG, Lareau DE, Hinricks JE, et al. Review article: soft tissue surgical procedure for optimizing anterior implant esthetics. International Journal of Dentistry. 2015: 1-9.

19. Bazzetti RG, Stahli A, Bazzetti MA, Sculean A. Soft tissue augmentation around osteointegrated and uncovered dental implants; a systematic review. Clinical Oral Investigation. 2017; 21: 53-70.

20. Salama H, Salama M, Garber D, Adar P. The interproximal height of bone: A guide post to predictable aesthetic strategies and soft tissue contours in anterior tooth replacements. Practical Periodontics and Aesthetic Dentistry. 1998; 10: 1131-1141.

21. Thoma DS, Buranawat B, Hammerle CHF, Held U, Jung RE. Efficacy of soft tissue augmentation around dental implant and in partially edentulous sites: a systematic review. Journal of Clinical Periodontology. 2014; 41: S77-S91.

22. Al-Hamdan KS. Esthetic soft tissue ridge augmentation around dental implant: Case report. Saudi Dental Journal. 2011; 23: 205-209.

23. Zetu I, Wang HL. Management of Interdental/inter implant papilla. Journal of Clinical Periodontology. 2005; 32: 831-839.

24. Gomez Meda R, Montoya Salazar V, Dalmau S, Torres Lagarese D. Three steps to maintaining predictable papilla and emergence profile in Immediate Implant placement. 3 year follow up case report. Journal of Clinical and Experimental Dentistry. 2018; 10: E513-519.

25. Rajguru SA, Tushar SP, Ashvini MP, Sachan K, Ishmani $\mathrm{SG}$, et al. Interimplant papilla reconstruction. Journal of Contemporary Dentistry. 2014; 4: 30-14.

26. Kan JYK, Rungcharassaeng K. Inter-implant papilla preservation in the esthetic zone. A report of six consecutive cases. International Journal of Periodontics and Restorative Dentistry. 2003; 23: 249-259.

27. Greenstein G, Tarnow D. Using papillae-sparing incisions in the esthetic zone to restore form and function. Compendium of Continuing Education. 2014: 315-322.
28. Kazor CE, Khalaf AS, Sarment DP, Misch CE, Wang HL. Implant plastic surgery: a review and rationale. Journal of Implantology. 2004; 30: 240-254.

29. Sclar AG. Strategies for management of single tooth extraction sites with esthetic implant therapy. Journal of Oral and Maxillofacial Surgery. 2004; 62: 90-105.

30. Salama H, Salama M. The role of orthodontic extrusion remodeling in enhancement of soft and hard tissue profiles prior to dental implant placement: A systematic approach to management of extraction site defects. International Journal of Periodontitics and Restorative Dentistry. 1993; 13: 312-333.

31. Hinds KF. Alveolar ridge development with forced Eruption and distraction of retained natural dentition. Oral and Maxillofacial Surgical Clinics of North America. 2004; 16: 75-89.

32. Rokn AR, Saffarpour A, Sadrimanesh R, Iranparavar A, Mahmoundzadeh $\mathrm{M}$, et al. Implant site by orthodontic forced eruption of non-treatable teeth: A case report. The Open Dentistry Journal. 2012; 6: 99-104.

33. Buser D, Duga K, Hirt HP, Schenk RK. Lateral ridge augmentation using autografts an barrier membranes: A clinical study of 40 partially edentulous patients. Journal of Oral Maxillofacial Surgery. 1996; 54: 420-432.

34. Goyal M, Mittal N, Gupta GK, Singhal M. Ridge augmentation in implant dentistry. Journal of the International Clinical Dental Research. 2015: 7: 94-112.

35. Milinkovic I, Cordaro L. Are there Specific indications for the alveolar bone augmentation procedures for dental implant placement? A systematic Review. International Journal of Oral and Maxillofacial Surgery. 2014; 43: 606-625.

36. Keestra JAJ, Barry O, Delong L, Wahl G. Long term effects of vertical bone augmentation: A systematic review. Journal of Applied Oral Science. 2016; 24: 3-17.

37. Palacci P, Nowzari H. Soft tissue enhancement around dental implants. Periodontology. 2000; 47: 113-132.

38. Tarnow DP, Cho Sc, Wallace SS. The effect of interImplant distance on the height of inter-implant bone crest. Journal of Periodontology. 2000; 71: 546-549.

39. Pradeep AR, Karthikeyan BV. Perimplant papilla reconstruction: Realities and limitations. Journal of Periodontology. 2006; 77: 534-544. 